\title{
A Fast Recursive Algorithm for System Identification and Model Reduction Using Rational Wavelets
}

\author{
Y. C. Pati* \\ Information Systems Laboratory \\ Dept. of Electrical Engineering \\ Stanford University, Stanford, CA 94305
}

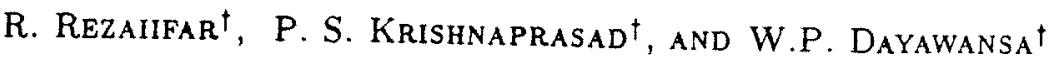 \\ Institute for Systems Research \\ Dept. of Electrical Engineering \\ University of Maryland, College Park, MD 20742
}

\begin{abstract}
In earlier work [Pati and Krishnaprasad 1992] it was shown that rational wavelet frame decompositions of the Hardy space $\mathrm{H}^{2}\left(\Pi^{+}\right)$may be used to efficiently capture time-frequency localized behavior of stable linear systems, for purposes of system identification and model-reduction. In this paper we examine the problem of efficient computation of low-order rational wavelet approximations of stable linear systems. We describe a variant of the Matching Pursuit algorithm [Mallat and Zhang 1992] that utilizes successive projections onto two-dimensional subspaces to construct rational wavelet approximants. The methods described here are illustrated by means of both simulations and experimental results.
\end{abstract}

\section{Introduction and Background}

It is well-known that rational functions play a central role in linear systems theory due to the equivalence of rational transfer functions and finitedimensional linear time-invariant (LTI) systems. In the context of linear system theory, rational approximation is the process of approximating an unparameterized, or high-dimensional (possibly infinitedimensional) LTI system by a finite-dimensional LTI

\footnotetext{
"This research was supported in part by NASA Headquarters, Center for Aeronautics and Space Information Sciences (CASIS) under Grant NAGW419,S6, and in part by the Advanced Research Projects Agency of the Department of Defense monitored by the Air Force Office of Scientific Rewearch under Contract F49620-93-1-0085.

This research was supported in part by the Air Force Office of Scientific Reseanch under contract F49620-92-J-0500, the AFOSR University Research Initiative Program under Grant AFOSR-90-0105, by the Army Research Office under Smart Structures URI Contract no. DAAL03-92-G-0121, and by the National Science Foundation's Engineering Research Centers Program, NSFD CDR 8803012.
}

system e.g. in problems of system identification and model-reduction. The goal of rational approximation in these problems is two-fold: (1) to accurately capture the relevant behavior of the underlying dynamical system, and (2) to keep the complexity (order) of the model as low as possible while meeting the first requirement.

Recently there has been a resurgence of interest in rational approximation methods using basis or basislike decompositions. In these methods it is assumed that the transfer function $G$ of interest maybe represented by a series of the form

$$
G(s)=\sum_{k} a_{k}(G) \Phi_{k}(s)
$$

where the $\Phi_{k}$ are fixed 'basis' functions (usually rational functions for finite $k$ ). Finite truncations of such series are then used as real-rational approximations to $G$. The two main advantages of these methods are (i) the model is linear in the parameters $a_{k}$, and (ii) it is often possible to incorporate various forms of $a$ priori knowledge into the approximation problem through suitable choice of the $\Phi_{k}(c . f .[3,6])$. Some examples of basis functions that have been considered in such applications are the Laguerre bases and Kautz filters (c.f. [6]) and more recently, rational wavelet bases, $[3,4]$.

In this paper we will consider the problem of efficiently computing rational approximations using the rational wavelet bases of Pati and Krishnaprasad $[3,4]$. A key property of such bases is that they form a set of time-frequency localized 'building blocks' with which a rational approximation may be constructed. One benefit of time-frequency localization is that it provides a useful vehicle for the incorporation of $a$ priori knowledge into parametric black-box models for system identification.

The rational bases constructed in $[3,4]$ are not bases in a strict sense, but rather a class of generalized bases, 
called frames, in the Hardy space $\mathrm{H}^{2}\left(\Pi^{+}\right)$. Representations of the form (1) where the $\Phi_{k}$ comprise a frame are not in general unique. Furthermore there is no 'natural' ordering of (affine) wavelet frames that suggests a particular truncation of (1) for any given transfer function $G(s)$. As our goal is to construct good approximations using as few terms as possible, we address the problem of: (a) selecting a truncation of the wavelet series expansion and (b) computing a parsimonious representation of any given transfer function, in an efficient recursive manner i.e. without computing the "complete" frame expansion of the transfer function. The algorithm we describe constructs the "best" one-term approximation of the error at each step and is a generalization of the Matching Pursuit (MP) algorithm [2]. The generalization is necessary to account for the real-rationality constraint on the approximants.

Experimental results from the identification of a flexible beam structure from measurements [5] and a second numerical example are used to illustrate the methods described here and a comparison is made with approximation using the Laguerre bases and the results in [3].

\subsection{Frames and Wavelets}

Frames (c.f. [1]) are natural generalizations of orthonormal bases in Hilbert spaces.

Definition 1.1 Given a Hilbert space $\mathcal{H}$ and a sequence of vectors $\left\{h_{n}\right\}_{n=-\infty}^{\infty} \subset \mathcal{H},\left\{h_{n}\right\}_{n=-\infty}^{\infty}$ is called a frame if there exist constants (frame bounds) $A>0$ and $B<\infty$ such that for every $f \in \mathcal{H}$,

$$
A\|f\|^{2} \leq \sum_{n}\left|<f, h_{n}>\right|^{2} \leq B\|f\|^{2} .
$$

A key property of frames is that the frame operator $\mathbf{S}$, defined by $\mathbf{S} f=\sum_{n}\left\langle f, h_{n}\right\rangle h_{n}, f \in \mathcal{H}$, is invertible and therefore any $f \in \mathcal{H}$ may be represented in terms of the frame elements:

$$
f=\sum<f, \mathbf{S}^{-1} h_{n}>h_{n}=\sum<f, h_{n}>\mathbf{S}^{-1} h_{n} .
$$

Affine, or wavelet frames, in the the Hilbert space $L^{2}(\mathbb{R})$ are frames in $L^{2}(\mathbb{R})$ constructed from dilates and translates of a single function $\psi$ (called the analyzing wavelet or mother wavelet) i.e. frames of the form $\left\{\psi_{m, n}(x)=a_{0}^{m / 2} \psi\left(a_{0}^{m} x-n b_{0}\right)\right\}$ where $a_{0}>0$ and $b_{0}$ are fixed constants. For such constants $a_{0}$ and $b_{0}$ to exist ${ }^{1} \psi$, must also satisfy the admissibility con-

\footnotetext{
${ }^{i}$ Numerically, $\alpha_{0}$ and $b_{0}$ may be determined by application of a theorem of Daubechies [1].
}

dition $\int_{\mathbb{R}}|\widehat{\psi}(\omega)|^{2} /|\omega| d \omega<\infty$, where $\widehat{\psi}$ is the Fourier transform of $\psi$. It is now well-known that wavelet frames may be constructed to form a set of functions that are 'well-localized' in time-frequency. Furthermore functions that exhibit time-frequency localized behavior may often be compactly represented in terms of wavelet 'bases'.

\subsection{The Hardy Space $\mathrm{H}^{2}\left(\Pi^{+}\right)$}

We consider the class of transfer functions contained in the Hardy space $\mathrm{H}^{2}\left(\Pi^{+}\right)$, where $\Pi^{+}$denotes, the half-plane $\Re e(s)>0$.

Definition 1.2 Given a function $F$ that is analytic in $\Pi^{+}, F$ is said to belong to $\mathrm{H}^{2}\left(\Pi^{+}\right)$if

$$
\sup _{x>0} \int_{-\infty}^{\infty}|F(x+i y)|^{2} d y<\infty
$$

By the Paley-Wiener theorem, elements of $\mathrm{H}^{2}\left(\Pi^{+}\right)$ may be identified with transfer functions of causal, input-output stable, linear time-invariant systems. The following notation is employed in the remainder of this paper:

$$
\begin{aligned}
\mathrm{H}_{\mathbf{R}}^{2}\left(\Pi^{+}\right)= & \begin{array}{l}
\text { Laplace transforms of real-valued } \\
\text { functions in } \mathrm{L}^{2}(0, \infty)
\end{array} \\
\mathrm{RH}^{2}\left(\Pi^{+}\right)= & \begin{array}{l}
\text { real-rational functions in } \mathrm{H}^{2}\left(\Pi^{+}\right), \\
\text {i.e. rational functions in } \mathrm{H}^{2}\left(\Pi^{+}\right) \\
\text {with real coefficients. }
\end{array}
\end{aligned}
$$

Thus $\mathrm{RH}^{2}\left(\Pi^{+}\right)\left(\subset \mathrm{H}_{\mathrm{R}}^{2}\left(\Pi^{+}\right)\right)$represents transfer functions of causal, finite-dimensional, linear systems with real-valued, square-integrable weighting patterns.

\subsection{WS Decompositions and Rational Ap- proximation}

In $[3,4]$ frames of rational wavelets in $\mathrm{H}^{2}\left(\Pi^{+}\right)$were used to construct rational approximations to transfer functions. These frames take the form $\left\{\Psi_{m, n}\right\}$, where the analyzing wavelet $\Psi$ is chosen to be real-rational ${ }^{2}$ $\left(\Psi \in \mathrm{RH}^{2}\left(\Pi^{+}\right)\right)$, and

$$
\Psi_{m, n}(s)=a_{0}^{m / 2} \Psi\left(a_{0}^{m} s-i n b_{0}\right), \quad a_{0}>0 .
$$

Note that given any transfer function in $\mathrm{H}_{\mathbf{R}}^{2}\left(\Pi^{+}\right)$, arbitrary truncations of its wavelet series expansion with respect to $\Psi \in \mathrm{RH}^{2}\left(\Pi^{+}\right)$are not in general realrational functions. The following theorem provides

\footnotetext{
${ }^{2}$ As in the cace of wavelets in $L^{2}(\mathbb{R})$, if $\Psi$ satisfies an appropriately defined admissibility condition (cf. [3]), it is possible to determine constants $a_{0}>0$ and $b_{0}$ such that the family $\left\{\Psi_{m, n}\right\}_{m, n \in Z}$, is a frame for $\mathrm{H}^{2}\left(\Pi^{+}\right)$.
} 
the necessary bridge between wavelet decompositions using a real-rational analyzing wavelet and a series representation in terms of real-rational functions.

Theorem 1.1 ([3]) Let $\Psi \in \mathrm{RH}^{2}\left(\Pi^{+}\right)$, be an admissible analyzing wavelet, such that $\left(\Psi, a_{0}, b_{0}\right)$ generates an affine frame $\left\{\Psi_{m, n}\right\}$ for $\mathrm{H}^{2}\left(\Pi^{+}\right)$. Then any $F$ in $\mathrm{H}_{\mathbf{R}}^{2}\left(\Pi^{+}\right)$may be represented as,

$$
F=\sum_{m, n \in \mathbf{Z}}\left\langle F, \mathbf{S}^{-1} \Psi_{m, n}\right\rangle \Psi_{m, n}=\sum_{m} \sum_{n=0}^{\infty} F^{m, n}
$$

where each $F^{m, n}$ is a real-rational function defined by,

$$
\begin{aligned}
& F^{m, 0}=\left\langle F, \mathbf{S}^{-1} \Psi_{m, 0}\right\rangle \Psi_{m, 0} \\
& F^{m, n}=\left\langle F, \mathbf{S}^{-1} \Psi_{m, n}\right\rangle \Psi_{m, n}+\overline{\left\langle F, \mathbf{S}^{-1} \Psi_{m, n}\right\rangle} \Psi_{m,-n}
\end{aligned}
$$

for $m \in \mathbf{Z},-n=1,2, \ldots$

The rightmost series in (6) is referred to as a wavelfft system (WS) decomposition of $F \in \mathrm{H}_{\mathbf{R}}^{2}\left(\Pi^{+}\right)$. Given a transfer function $F \in \mathrm{H}_{\mathbf{R}}^{2}\left(\Pi^{+}\right)$, and its WS decomposition, a real-rational approximation $\widetilde{F}$, to $F$, may be constructed as a truncated WS series, i.e

$$
\tilde{F}(s)=\sum_{(m, n) \in \mathcal{J}} F^{m, n}(s),
$$

where $\mathcal{J}$ is a suitably chosen finite index set, and $F^{m, n}$, are real-rational functions as in Theorem 1.1.

In $[3,4]$, the index set $\mathcal{J}$, was selected to include terms from the WS decomposition corresponding to the largest wavelet frame coefficients $\alpha_{m, n}=$ $\left\langle F, \mathbf{S}^{-1} \Psi_{m, n}\right\rangle$. As mentioned earlier, the drawbacks with this approach are that: (a) the frame expansion with respect to a large collection of vectors must first be computed, and (b) the frame expansion coefficients given by (3) do not necessarily generate the most parsimonious representation possible with respect to a particular collection of vectors.

\section{WS Rational Approximation via Successive Projections (WRASP)}

In the Matching Pursuit algorithm of Mallat and Zhang [2] approximations are generated recursively via a sequence of projections onto one-dimensional spaces. In particular, at each step one projects the current residual (error) onto each ('basis') vector individually and then selects the projection with maximum norm to be added to the current approximation. In the case of WS approximations additional constraints are imposed on the problem by the requirement of real-rational approximants. Here a generalized version of the matching pursuit algorithm may be applied by considering instead projections onto both one and two-dimensional subspaces. Let us define the following orthogonal projection operator $\left(\right.$ in $\left.\mathrm{H}^{2}\left(\Pi^{+}\right)\right)$:

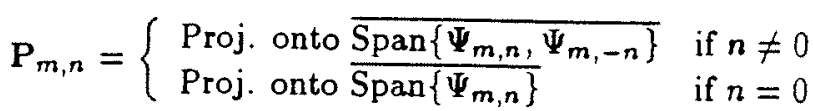

Note that for $n \neq 0, \mathbf{P}_{m, n}$ is a projection onto a two-dimensional subspace of $\mathrm{H}^{2}\left(\Pi^{+}\right)$. Now consider the following algorithm that we will refer to as the WRASP algorithm (WS Rational Approximation via Successive Projections). $F_{k}$ and $\mathbf{R}_{k} F$ denote the ap-proximation and the residual at the $k^{\text {th }}$ iteration respectively i.e. $F=F_{k}+\mathbf{R}_{k} F$.

\section{The WRASP Algorithm}

Initialization:

$$
F_{0}=0, \mathbf{R}_{0} F=F, k=0
$$

(I) Compute all projections

$$
\mathbf{P}_{m, n} \mathbf{R}_{k} F, \quad m \in \mathbf{Z}, n \in \mathbb{Z}^{+},
$$

where $\mathbf{Z}^{+}$denotes nonnegative integers.

(II) Find the 'largest' projection i.e. find $\left(m_{k}, n_{k}\right)$ such that,

$$
\left\|\mathbf{P}_{m_{k}, n_{k}} \mathbf{R}_{k} F\right\| \geq \beta \sup _{m \in \mathbf{Z}, n \in \mathbf{Z}^{+}}\left\|\mathbf{P}_{m, n} \mathbf{R}_{k} F\right\|,
$$

where $0<\beta \leq 1$.

(III) Update the model and residual:

$$
\begin{aligned}
F_{k+1} & =F_{k}+\mathbf{P}_{m_{k}, n_{k}} \mathbf{R}_{k} F \\
\text { and } \mathbf{R}_{k+1} F & =\mathbf{R}_{k} F-\mathbf{P}_{m_{k}, n_{k}} \mathbf{R}_{k} F
\end{aligned}
$$

(IV) Increment $k$ (i.e. $k \leftarrow k+1$ ) and repeat Steps (I)-(IV), until some stopping criterion has been satisfied.

The distinction between the above algorithm and the Matching Pursuit algorithm is in the form of the projections (c.f. (7)). 


\subsection{Some Properties WRASP}

As we are interested in real-rational approximants, we need to verify that the approximants $F_{k}$, at each step $k<\infty$ are real-rational and that they converge to $F$ as $k \rightarrow \infty$. We state this in the following theorem. A detailed proof of this theorem is omitted to conserve space and will appear elsewhere.

Theorem 2.1 Let $\left\{\Psi_{m, n}\right\}_{m, n \in Z}$ be a rational wavelet frame for $\mathrm{H}^{2}\left(\Pi^{+}\right)$, and let $\mathcal{I}$ be a subset of $\mathbf{Z}^{2}$, such that $(m, n) \in \mathcal{I} \Leftrightarrow(m,-n) \in \mathcal{I}$. Also let $V={\overline{\operatorname{Span}\left\{\Psi_{m, n}\right\}_{(m, n) \in I}}}$ and let $P_{V}$ the denote orthogonal projection operator onto $\mathrm{V}$. Then for any $F \in \mathrm{H}_{\mathbf{R}}^{2}\left(\Pi^{+}\right)$.

(1) $\left\|\mathbf{R}_{k} F-\mathbf{P}_{V \perp} F\right\| \longrightarrow 0$ as $k \rightarrow \infty$

(2) $\mathbf{P}_{V} F=\sum_{k=0}^{\infty} \mathbf{P}_{m_{k}, n_{k}} \mathbf{R}_{k} F$.

(3) $F_{N}=\sum_{k=0}^{N} \mathbf{P}_{m_{k}, n_{k}} \mathbf{R}_{k} F \in \mathrm{RH}^{2}\left(\Pi^{+}\right), \quad N<\infty$.

(4) $\left\|\mathbf{P}_{V} F\right\|^{2}=\sum_{k=0}^{\infty}\left\|\mathbf{P}_{m_{k}, n_{k}} \mathbf{R}_{k} F\right\|^{2}$.

Proof: The proof of this theorem approximately parallels the proof of Theorem 1 in [2], with onedimensional projections replaced by the $\mathbf{P}_{m, n}$. Realrationality of the approximants $F_{N}$, follows from Lemma 2.1 below.

\subsubsection{Computational Aspects of WRASP}

First of all note that the inner products $\left\{\left\langle F, \Psi_{m, n}\right\rangle\right\}_{m, n}$ may be readily computed by convolution with $\widetilde{\mathbf{\Psi}}_{m}=a_{0}^{m / 2} \mathbf{\Psi}\left(-a_{0}^{m} \cdot\right)$ followed by sampling at each dilation level $m$ i.e.

$$
\left\langle F, \Psi_{m, n}\right\rangle=\left(F * \tilde{\Psi}_{m}\right)\left(a_{0}^{-m} b_{0} n\right)
$$

Furthermore the projections $\mathbf{P}_{m, n} F$ are easily obtained using Equation (10) of the following lemma.

Lemma 2.1 Given any function $F \in \mathrm{H}_{\mathbf{R}}^{2}\left(\Pi^{+}\right)$, the projections $\mathbf{P}_{m, n} F$, are real-rational and

$\mathbf{P}_{m, n} F$

$=\alpha^{2}\left[\left(\left\langle F, \Psi_{m, n}\right\rangle-\overline{\left\langle F, \Psi_{m, n}\right\rangle}\left\langle\Psi_{m,-n}, \Psi_{m, n}\right\rangle\right) \Psi_{m, n}\right.$

$\left.+\left(\overline{\left\langle F, \Psi_{m, n}\right\rangle}-\left\langle F, \Psi_{m, n}\right\rangle \overline{\left\langle\Psi_{m,-n}, \Psi_{m, n}\right\rangle}\right) \Psi_{m,-n}\right]$.

for $n \neq 0$, where, $\alpha^{2}=\left(1-\left|\left\langle\Psi_{m,-n}, \Psi_{m, n}\right\rangle\right|^{2}\right)^{-1}$. For $n=0, \mathbf{P}_{m, n} F=\left\langle F, \Psi_{m, n}\right\rangle \Psi_{m, n}$.
For $k>0, \mathbf{R}_{k+1} F$ may be computed from $\mathbf{R}_{k} F$ using the recursion,

$$
\begin{aligned}
& \left\langle\mathbf{R}_{k+1} F, \Psi_{m, n}\right\rangle \\
& \quad=\left\langle\mathbf{R}_{k} F, \Psi_{m, n}\right\rangle-c_{k}\left\langle\Psi_{m_{k+1}, n_{k+1}}, \Psi_{m, n}\right\rangle \\
& -\overline{c_{k}}\left\langle\Psi_{m_{k+1},-n_{k+1}}, \Psi_{m, n}\right\rangle
\end{aligned}
$$

where $c_{k}$ is the coefficient obtained from the previous projection $\mathbf{P}_{m_{k+1}, n_{k+1}} \mathbf{R}_{k} F$, i.e. $\mathbf{R}_{k} F=$ $c_{k} \Psi_{m_{k+1}, n_{k+1}}+\overline{c_{k}} \boldsymbol{\Psi}_{m_{k+1},-n_{k+1}}+\mathbf{R}_{k+1} F$. Hence the computation required for WRASP takes a simple recursive form just as the computation required for Matching Pursuit. Also the starting point for the recursions in both algorithms are the inner products $\left\{\left\langle F, \Psi_{m, n}\right\rangle\right\}_{m, n}$, (computed using (8)), and the (Gram) matrix $\mathbf{A}=\left[a_{i j}\right]=\left[\left\langle\Psi_{m_{i}, n_{i}}, \Psi_{m_{j}, n_{j}}\right\rangle\right]$ (precomputed for the dictionary).

\section{Examples}

In the following examples, the analyzing wavelet is taken to be, $\Psi(s)=\frac{1}{(s+25)^{2}+1}$, which generates an affine frame for $\mathrm{H}^{2}\left(\Pi^{+}\right)$with $a_{0}=2,0<b_{0}<16.5$.

Example I: In the first example we consider the problem of approximating an unparameterized model of a single channel of a cochlear filterbank (c.f. [3]) by a finite-dimensional system. Figure 1 compares the approximation performance of the WRASP algorithm applied to this problem with two other rational approximation methods: (i) rational wavelet approximation using the largest coefficients of a 'full' decomposition, and (ii) rational approximation using the Laguerre basis.

Example II: In this example the WRASP algorithm is used construct an approximate finitedimensional model for a flexible beam apparatus equipped with piezoceramic sensors/actuators using measured data [5]. The experimental setup is shown in Figure 2. Figure 3 compares WRASP with Laguerre basis approximation and Figure 4 compares the identified WRASP model with the measured response.

\section{Summary and Discussion}

In this paper we have described a recursive algorithm, which we refer to as WRASP (WS Rational 


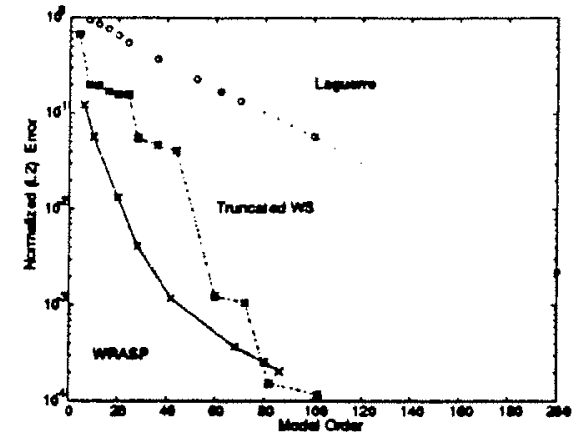

Figure 1: Example I (Cochlear Filter): Normalized $\left(\mathrm{H}^{2}\left(\Pi^{+}\right)\right)$approximation error versus model-order: $(x)$ the WRASP algorithm, (*) rational wavelet approximation using the full decomposition, and (o) Laguerre basis approximation.

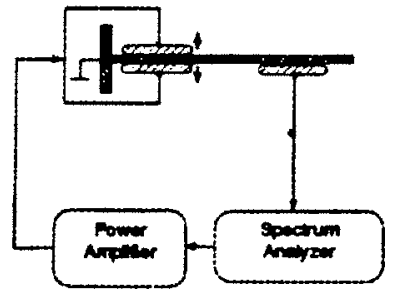

Figure 2: Experimental flexible beam setup. The power amplifier drives a pair of piezoceramic actuators bonded to the beam and a spectrum analyzer measures the response through a piezoceramic sensor.

Approximation via Successive Projections), to effciently compute low-order rational wavelet approximations of transfer functions. Convergence and computational aspects of WRASP were discussed and the algorithm was applied to two examples of system identification/model-reduction problems.

\section{References}

[1] Ingrid Daubechies. The wavelet transform, timefrequency localization and signal analysis. IEEE Transactions on Information Theory, 36(5):9611005, September 1990.

[2] S. Mallat and Z. Zhang. Matching pursuits with time-frequency dictionaries. Preprint. Submitted to IEEE Transactions on Signal Processing, 1992.

[3] Y. C. Pati. Wavelets and Time-Frequency Methods in Linear Systems and Neural Networks. PhD thesis, University Of Maryland, College Park, MD, 1992.

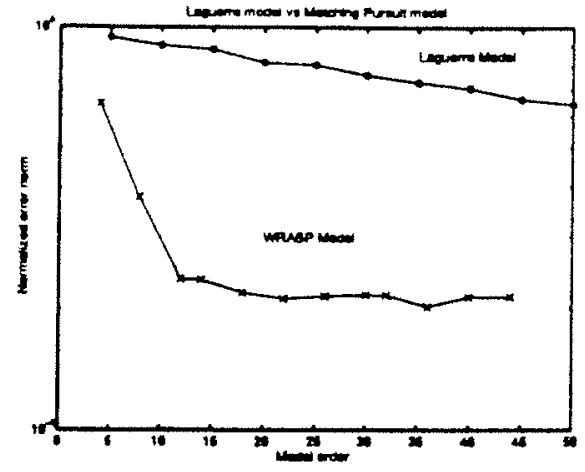

Figure 3: Example II (Flexible beam): Normalized $\left(\mathrm{H}^{2}\left(\Pi^{+}\right)\right)$approximation error versus model-order: $(x)$ the WRASP algorithm, and (o) Laguerre basis approximation.
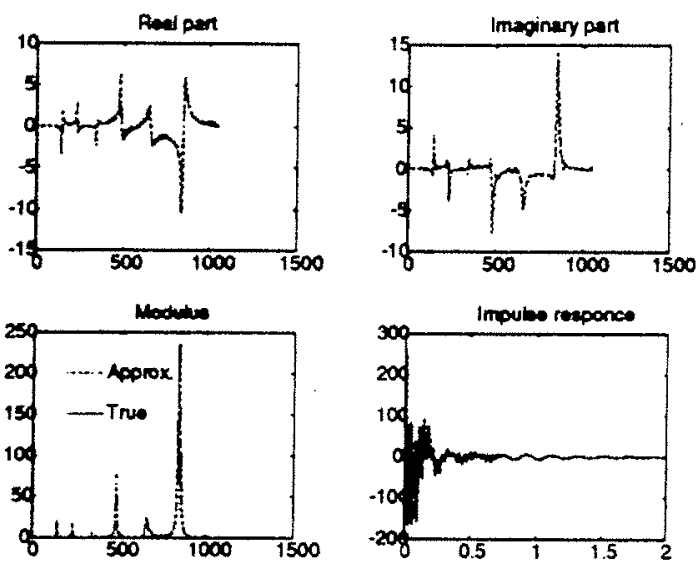

Figure 4: Example II (Flexible beam): WRASP approximation results comparing measured and approximate models. Model order $=46$

[4] Y. C. Pati and P. S. Krishnaprasad. Rational wavelets in approximation and identification of stable linear systems. In Proceedings 31st IEEE Conference on Decision and Control, pages 15021507, N.Y., December 1992. IEEE.

[5] R. Rezaiifar. Smart structures and wavelet based system identification. Master's thesis, University of Maryland, College Park, MD, 1993. Institute for Systems Research Technical Report No. MS 93-18.

[6] Bo Wahlberg. System identification using Laguerre models. IEEE Transactions on Automatic Control, 36(5):551-562, 1991. 